\title{
A REMARK ON THE EXTENSION OF THE CONCEPT OF INCIDENCE ALGEBRAS TO NONLOCALLY FINITE PARTIALLY ORDERED SETS
}

\author{
BONIFACE I. EKE
}

\author{
Received 4 March 2003
}

An incidence algebra of a nonlocally finite partially ordered set $Q$ is a very rare concept, perhaps nonexistent. In this note, we will attempt to construct such an algebra.

2000 Mathematics Subject Classification: 06A11.

1. Introduction. Let $P$ be a partially ordered set (poset) and $K$ a field of characteristic 0 . The functions $f: P \times P \rightarrow K$, such that $x \nless y$ implies $f(x, y)=0$, are called the incidence functions of $P$ over $K$. The set of such functions is denoted by $\vartheta(K, P)$. $P$ is called locally finite if for every $x, y \in P$ the interval $[x, y]=\{t \in P \mid x \leq t \leq y\}$ is finite. When $P$ is locally finite, $\vartheta(K, P)$ becomes a $K$-algebra under a multiplication $(*)$ defined by convolution:

$$
f * g(x, y)=\sum_{x \leq t \leq y} f(x, t) g(t, y)
$$

and the algebra $\mathcal{Y}(K, P)$ is called the incidence algebra of $P$ over $K[1,2]$.

If $P$ is not locally finite, the expression (1.1) may not make sense. So, one does not often hear of an incidence algebra of a nonlocally finite poset. Our purpose in this note is to show that if $Q$ is any nonlocally finite poset and $P$ is a locally finite poset, we can form a nonlocally finite poset $Q S(P)$ for which an incidence algebra $9(K, Q S(P))$ can be constructed.

Moreover, the posets $Q$ and $P$ are both embeddable in $Q S(P)$, while the set $9(K, Q)$ and the algebra $9(K, P)$ are both embeddable in $9(K, Q S(P))$, and if $|P| \leq|Q|$, then $|Q S(P)|=|Q|$. Besides, for the fixed posets $P$ and $Q$, the incidence algebra $\vartheta(K, Q S(P))$ is unique up to isomorphism. All these are established in Section 2.

In Section 3, we isolate the auxiliary locally finite poset $P$ and try to deal directly with $Q$. However, because of the problem still posed by (1.1), we can only construct a sequence of what are called truncated incidence algebras for the nonlocally finite poset $Q$. For this purpose, we will need an additional hypothesis that $Q$ is well ordered.

2. The construction of $Q S(P)$ and $\mathcal{Y}(K, Q S(P))$. We will assume throughout that $P$ is a locally finite poset, $Q$ a nonlocally finite poset, and $K$ is a field of characteristic 0 . Let $Q S(P)$ be the Cartesian product $P \times Q$. We will denote the order relation in $P$ by $\leq^{(1)}$ and the order relation in $Q$ by $\leq^{(2)}$. Then we define an order relation $\leq$ in $Q S(P)$ by 
$(x, r) \leq(y, s)$ if and only if $x \leq^{(1)} y$ and $r \leq^{(2)} s$. It is clear that, with the relation $\leq$, $Q S(P)$ is a partially ordered set. However, $Q S(P)$ is not locally finite.

We will define addition and scalar multiplication on $\vartheta(K, Q S(P))$ as in [1]. We now need to define the convolution multiplication shown in (1.1) on $\mathcal{\vartheta}(K, Q S(P))$ so that it will make sense.

For a fixed $r \in Q$, denote $P \times\{r\}$ by $P_{r}$. If $(x, r)$ and $(y, s)$ are any two elements of $Q S(P)$, then $(x, r) \in P_{r}$, while $(y, s) \in P_{s}$. Moreover, $P_{r}$ and $P_{s}$ are locally finite subposets of $Q S(P)$. Denote $(x, r)$ by $u$ and $(y, s)$ by $v$, and let $T=\left\{t \in P \mid x \leq^{(1)}\right.$ $\left.t \leq{ }^{(1)} y\right\}$. Then the set $T(u, v)=(T \times\{r\}) \cup(T \times\{s\}) \subseteq Q S(P)$ is finite. Let $J(u, v)=$ $[u, v] \cap T(u, v)$. We define the operation $(*)$ on $\vartheta(K, Q S(P))$ by the following: for all elements $u$ and $v$ in $Q S(P)$ and for all $f, g$ in $\mathcal{\vartheta}(K, Q S(P))$,

$$
f * g(u, v)=\sum_{z \in J_{(u, v)}} f(u, z) g(z, v)
$$

Clearly, (2.1) is now well defined. The associativity follows from [1, Proposition 4.1]. Consequently, with (2.1), $\vartheta(K, Q S(P))$ is an incidence algebra of $Q S(P)$ over $K$.

$P$ is isomorphic to $P_{r}$ for each $r \in Q$. Similarly, for each $y \in P, Q$ is isomorphic to $Q_{y}=\{y\} \times Q$. Hence both $P$ and $Q$ are embeddable in $Q S(P)$. Moreover, the correspondence $\mu_{r}: f \mapsto f_{r}$, where $f_{r}$ is defined by $f_{r}\left(x_{r}, y_{r}\right)=f(x, y)$, is an isomorphism of $\vartheta(K, P)$ onto $\vartheta\left(K, P_{r}\right)$. Consequently, $\vartheta(K, P)$ is embeddable in $\vartheta(K, Q S(P))$. By a similar device, we find that $\vartheta(K, Q)$ is also embeddable in $\vartheta(K, Q S(P))$. For the uniqueness of $\vartheta(K, Q S(P))$, we will prove the following.

Proposition 2.1. If $P^{\prime}$ and $Q^{\prime}$ are any posets such that $P$ is isomorphic to $P^{\prime}$ and $Q$ is isomorphic to $Q^{\prime}$, then $9(K, Q S(P))$ is isomorphic to $9\left(K, Q^{\prime} S\left(P^{\prime}\right)\right)$.

Proof. Let $\sigma: P \rightarrow P^{\prime}$ be an isomorphism while $\theta: Q \rightarrow Q^{\prime}$ is an isomorphism. Define $\eta: Q S(P) \rightarrow Q^{\prime} S\left(P^{\prime}\right)$ by $\eta(x, r)=(\sigma(x), \theta(r))$. If $\eta(x, r)=\eta(y, s)$, then $(\sigma(x), \theta(r))=$ $(\sigma(y), \theta(s))$. By the definition of the order relation in $Q^{\prime} S\left(P^{\prime}\right)$, we must have $\sigma(x)=$ $\sigma(y)$ and $\theta(r)=\theta(s)$. Consequently, $x=y$ and $r=s$. Hence, $(x, r)=(y, s)$. This shows that $\eta$ is injective. Clearly, also $\eta$ is surjective. Therefore, $\eta$ is an isomorphism. For each $u \in Q S(P)$, denote $\eta(u)$ by $u^{\prime}$. Now define $\beta: \vartheta(K, Q S(P)) \rightarrow \vartheta\left(K, Q^{\prime} S\left(P^{\prime}\right)\right)$ by $\beta(f)=f^{\prime}$, where $f^{\prime}$ is defined by $f^{\prime}\left(u^{\prime}, v^{\prime}\right)=f(u, v)$ for all $u^{\prime}, v^{\prime} \in Q^{\prime} S\left(P^{\prime}\right)$. One can directly check that $\beta$ is also an isomorphism. Hence, the proposition holds.

We observe that for the locally finite poset $P$, one could have chosen any nonempty finite subset of $Q$ itself. We will call the algebra $\vartheta(K, Q S(P))$ the incidence algebra of $Q$ relative to $P$.

3. Truncated incidence algebras. Our interest now is to see what we can achieve by isolating the locally finite poset $P$ and dealing directly with $Q$. However, (1.1) still poses a problem. Nevertheless, following the motivation received from Section 2, what we need is to try to use a finite number of elements of the interval $[r, s]$ at a time, for any two elements $r$ and $s$ of the nonlocally finite poset $Q$. Then arises the question of how to choose the finite number of elements from $[r, s]$. The formula for choosing such elements is outlined below for the case where $Q$ is well ordered. What makes it possible 
is the property of a well-ordered set whereby not only does every nonempty subset of such a set have a first element, but also such a first element is unique [3, Theorems 64 and 65, page 76]. First, we show the existence of a well-ordered nonlocally finite poset $Q$.

EXAMPLE 3.1. Let $Q=\{1 / n \mid n \in \mathbb{N}\} \cup\{O\}$, where $\mathbb{N}$ is the set of natural numbers. $Q$ is a poset subject to the usual relation " $\geq$ " (greater than or equal to). Clearly, also $Q$ is well ordered by " $\geq$ ". However, for any $a \in Q, a \neq 0$, the interval $[0, a]$ is infinite. Hence, $Q$ is not locally finite.

Now let $W$ be any well-ordered poset and let $r \leq s \in W$. Set $W_{0}=[r, s]$. Let $W_{1}=$ $W_{0}-\{r\}$. Then, if $W_{1} \neq \varnothing, W_{1}$ has a unique first element $t_{1}$. Let $W_{2}=w_{1}-\left\{t_{1}\right\}$. If $W_{2} \neq \varnothing$, then $W_{2}$ has a unique first element $t_{2}$. In general, $W_{i}=W_{i-1}-\left\{t_{i-1}\right\}$, where $t_{i-1}=$ first element of $W_{i-1}$, and $t_{0} \equiv r$.

For any fixed natural number $n$, let $T_{n}(r, s)=\left\{r, t_{1}, \ldots, t_{n}, s\right\}$. Let

$$
J_{n}(r, s)= \begin{cases}{[r, s]} & \text { if }[r, s] \text { is finite, } \\ T_{n}(r, s) & \text { otherwise. }\end{cases}
$$

Then define the convolution multiplication $*$ on $\mathcal{\vartheta}(K, W)$ by the following: for all $r, s \in$ $W$ and for all $f, g \in \mathcal{G}(K, W)$,

$$
f * g(r, s)=\sum_{t \in J_{n}(r, s)} f(r, t) g(t, s) .
$$

Subject to (3.2), $9(K, W)$ is an incidence algebra. We denote this incidence algebra by $\vartheta_{n}(K, W)$, and $\vartheta_{n}(K, W)$ is called a truncated incidence algebra of $W$ over $K$.

It is clear that $T_{n}(r, s) \subseteq T_{n+1}(r, s)$ for all $n \in \mathbb{N}$. We will call the incidence algebra $\vartheta_{n+1}(K, W)$ a refinement of the incidence algebra $\vartheta_{n}(K, W)$. The sequence $\left\{\vartheta_{n}(K, W)\right\}$ of incidence algebras is finite if and only if $W$ is locally finite.

We now observe that a well-ordered nonlocally finite poset $Q$ is associated with an infinite sequence of truncated incidence algebras, where each is a nontrivial refinement of the one before it. Unifying these algebras to form one incidence algebra of $Q$ over $K$ remains an open problem.

\section{REFERENCES}

[1] M. Aigner, Combinatorial Theory, Grundlehren der mathematischen Wissenschaften, vol. 234, Springer-Verlag, New York, 1979.

[2] R. P. Stanley, Structure of incidence algebras and their automorphism groups, Bull. Amer. Math. Soc. 76 (1970), 1236-1239.

[3] P. Suppes, Axiomatic Set Theory, Dover Publications, New York, 1972.

Boniface I. Eke: Department of Mathematics, Morgan State University, Baltimore, MD 21251, USA

E-mail address: beke@morgan .edu 


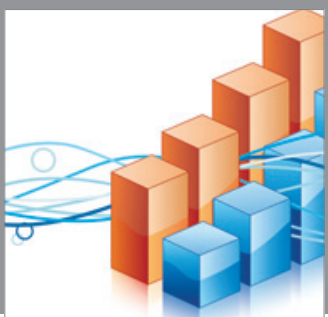

Advances in

Operations Research

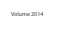

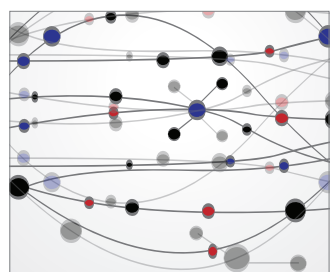

\section{The Scientific} World Journal
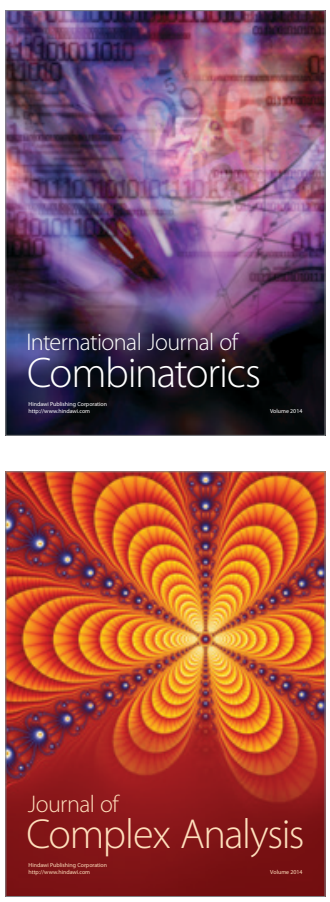

International Journal of

Mathematics and

Mathematical

Sciences
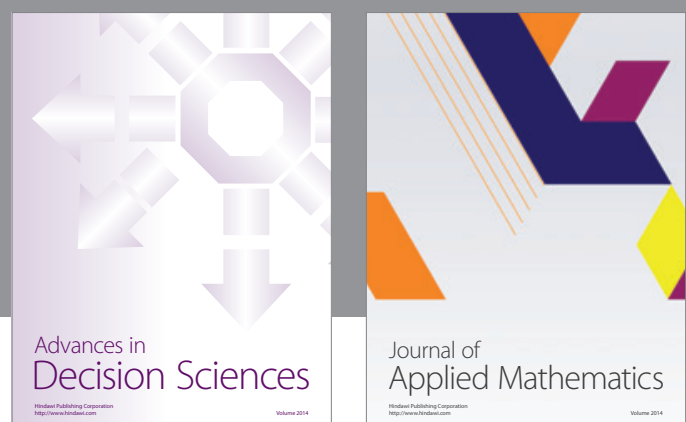

Journal of

Applied Mathematics
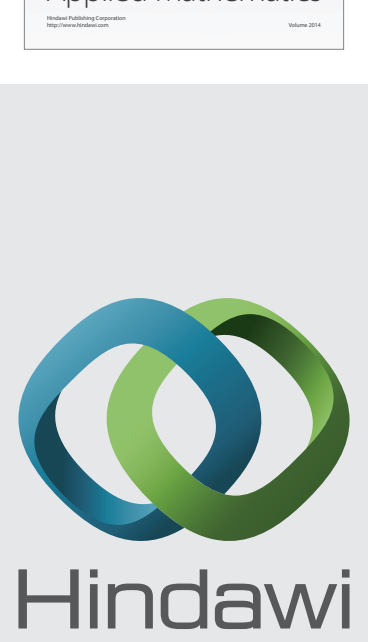

Submit your manuscripts at http://www.hindawi.com
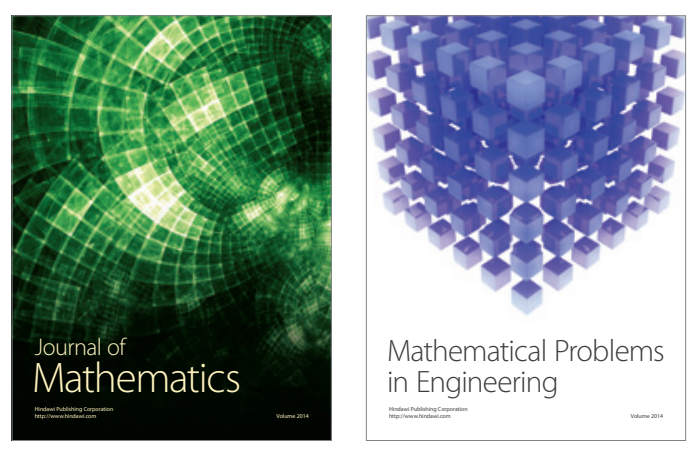

Mathematical Problems in Engineering
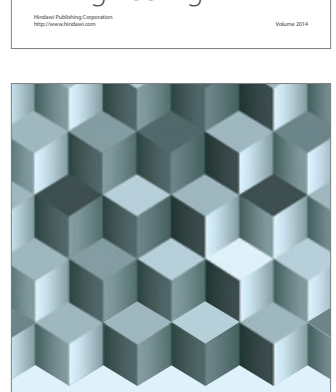

Journal of

Function Spaces
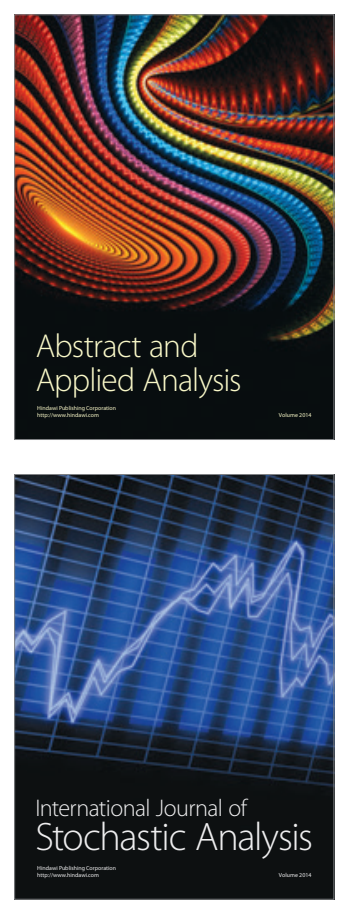

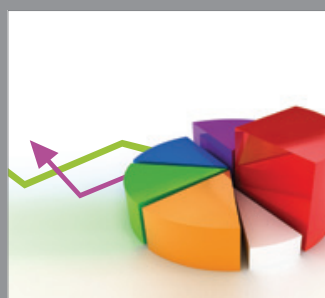

ournal of

Probability and Statistics

Promensencen
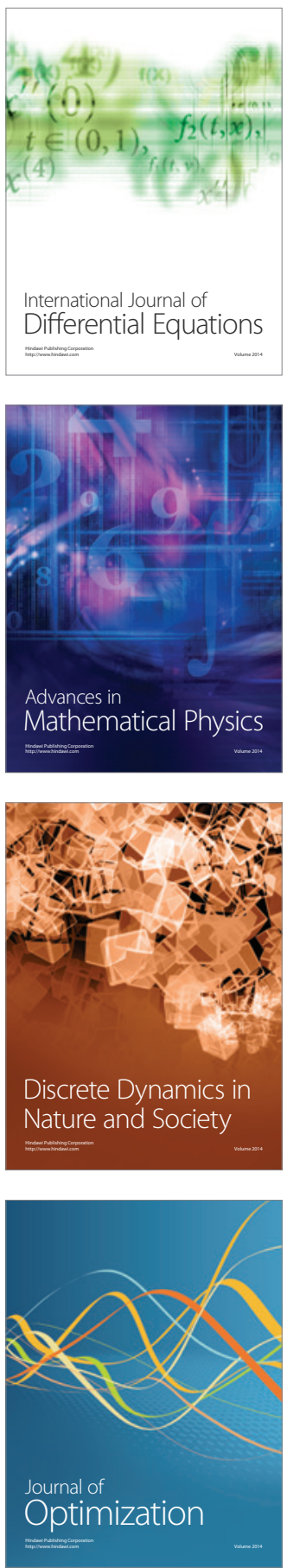